\title{
Retraso del desarrollo del lenguaje en niños de 24 meses en un centro de salud en la Ciudad de Buenos Aires
}

\author{
Language development delay in 24-month-old children at a \\ health care center of the City of Buenos Aires
}

\author{
Dr. Alejandro Blumenfeld, ${ }^{a}$ Dr. José Carrizo Olalla, ${ }^{b}$ Dra. Sabrina I. D’Angelo, ${ }^{c}$ \\ Dra. Natalia S. González, ${ }^{c}$ Dra. Yael Sadras, ${ }^{c}$ Lic. Sandra Graizer, ${ }^{d}$ \\ Dr. Alejandro Macario, ${ }^{a}$ y Dra. Graciela Salamanco ${ }^{e}$
}

\section{RESUMEN}

Introducción. El retraso del desarrollo del lenguaje representa un desafío frecuente para pediatras y otros profesionales. El objetivo principal fue establecer su prevalencia en niñas y niños usuarios de un centro de salud.

Población y métodos. Se evaluó el vocabulario expresivo de niñas y niños de 24 meses en un centro de salud utilizando el Inventario de Desarrollo de Habilidades Comunicativas MacArthur-Bates, versión breve (adaptación argentina). Se investigó la asociación entre el retraso del lenguaje y las características demográficas, socioeconómicas, conductuales / emocionales y de estrés parental. En aquellos con retraso expresivo, se evaluó el vocabulario receptivo y se investigaron posibles causas subyacentes (retraso cognitivo no verbal, trastornos del espectro autista, patología del oído medio e hipoacusia).

Resultados. Se observó un retraso del desarrollo del lenguaje en 16 de 138 participantes (11,6\%; intervalo de confianza -IC- 95\%: 6,2-17\%), asociado significativamente con antecedentes familiares de retraso del lenguaje, estrés parental y comportamiento problemático. El compromiso del vocabulario receptivo se identificó en 13 de 16 casos con retraso del desarrollo del lenguaje, y 7 presentaron sospecha de trastorno del espectro autista, de retraso global del desarrollo o ambas. Se observó la patología del oído medio en 5 de 9 estudiados. Los 9 participantes a los que se realizó una audiometría no presentaron resultados patológicos de acuerdo con los criterios adoptados en el presente trabajo.

Conclusiones. El retraso del desarrollo del lenguaje representa un problema prevalente en nuestra población y se asoció principalmente con problemas de comportamiento y antecedentes familiares de retraso del lenguaje.

Palabras clave: retraso en el desarrollo del lenguaje oral, atención primaria de salud, desarrollo infantil, trastorno del espectro autista, hipoacusia.

http:/ / dx.doi.org/10.5546/ aap.2018.242

Texto completo en inglés:

http:/ / dx.doi.org/10.5546/ aap.2018.eng.242
Cómo citar: Blumenfeld A, Carrizo Olalla J, D'Angelo SI, et al. Retraso del desarrollo del lenguaje en niños de 24 meses en un centro de salud en la Ciudad de Buenos Aires. Arch Argent Pediatr 2018;116(4):242-247.

\section{INTRODUCCIÓN}

Desde la infancia temprana, los niños desarrollan pautas de lenguaje de complejidad progresiva, siguiendo una secuencia predecible que comienza por vocalizaciones, sílabas y luego palabras. ${ }^{1}$ Según el modelo ecológico, este desarrollo está determinado por factores biológicos, hereditarios, psicológicos y socioeconómicos. ${ }^{2} \mathrm{Si}$ bien el término "retraso del desarrollo del lenguaje" carece de una definición específica, en la literatura científica, se emplea universalmente para identificar a niños y niñas de 18-36 meses de edad que no alcanzan las pautas mínimas de vocabulario expresivo esperables para su edad y género. Son sinónimos "retraso en la adquisición del lenguaje" y "retraso temprano del lenguaje". ${ }^{3}$ También se encuentra muy difundido en la literatura el término más coloquial de "hablantes tardíos" (en inglés: late talkers). ${ }^{4}$

No se han encontrado estudios en Argentina sobre la prevalencia del retraso del lenguaje. En estudios de otros países donde se abordaron poblaciones de niveles socioeconómicos predominantemente medio y alto, la prevalencia se ubicó entre 10 y 20\%..$^{5-8} \mathrm{Si}$ bien el pronóstico de estos niños es, por lo general, bueno, se ha estimado que 
aproximadamente un tercio presenta compromiso adicional del vocabulario receptivo, lo que incrementa el riesgo de problemas del lenguaje en etapas posteriores de la infancia. ${ }^{9}$ Además, existe un subconjunto de niños que presenta causas subyacentes de retraso del lenguaje, como, por ejemplo, síndromes genéticos, discapacidad intelectual, trastornos del espectro autista (TEA), hipoxia perinatal o hipoacusia. ${ }^{1}$

El objetivo del presente estudio fue determinar la prevalencia del retraso del desarrollo del lenguaje en niños/as de 24 meses en un centro de salud de la Ciudad Autónoma de Buenos Aires (CABA) y los factores asociados.

\section{POBLACIÓN Y MÉTODOS}

Esta investigación se realizó entre febrero de 2015 y octubre de 2016 en el Centro de Salud y Acción Comunitaria (CeSAC) $\mathrm{n}^{\circ} 44$, un efector público del primer nivel de atención ubicado en el sur de la CABA. En las evaluaciones de causas subyacentes, participaron también el Servicio de Otorrinolaringología del Hospital General de Agudos "Parmenio Piñero" y el Servicio de Audiología del Hospital de Rehabilitación "Manuel Rocca", también de la CABA.

Se utilizó un diseño observacional analítico de corte transversal. Se incluyeron niños y niñas de 24 meses de edad que contaran con historia clínica en el CeSAC 44. Los criterios de exclusión fueron hipoacusia, problemas del desarrollo o TEA previamente diagnosticados, síndromes asociados a discapacidad intelectual (ej.: síndrome de Down), parálisis cerebral, patología perinatal que hubiera requerido ventilación mecánica o exanguinotransfusión, prematuros o de bajo peso al nacer (menores de 2500 gramos), antecedente de meningitis, convulsiones sin fiebre o epilepsia, neurocirugía, cardiopatías congénitas, patología oncológica y la pertenencia a hogares no hispanoparlantes o bilingües (así considerados si, al menos, uno de los padres hablaba diariamente otro idioma distinto del español). Estos criterios fueron usados en otros trabajos y permitieron determinar la prevalencia del retraso del lenguaje en niños y niñas aparentemente sanos. ${ }^{6-8,10}$

Se utilizó un muestreo por conveniencia, y se incorporaron consecutivamente todos los participantes disponibles. La convocatoria se realizó por contacto telefónico y se citó a uno de los padres. De manera complementaria, se dejaron notas de invitación en los domicilios ubicados a menos de 1000 metros del CeSAC 44 y se utilizaron carteles en la sala de espera e invitaciones en forma personal.

Las investigaciones se dividieron en 2 fases (Figura 1). En la fase 1, se estudiaron todos los participantes. La variable principal de resultado fue el vocabulario expresivo, evaluado mediante el instrumento Inventario de Desarrollo de Habilidades Comunicativas MacArthurBates. Palabras y Enunciados, versión breve (adaptación argentina) (Spanish-language MacArthur-Bates Communicative Development Inventories short form II, S-CDI SF-II). Este instrumento fue autoadministrado por uno de los padres y consistía en identificar de una lista preseleccionada de 100 palabras aquellas que el niño usaba regularmente. ${ }^{11}$ Fue validado en México, y, para este estudio, 12 de las 100 palabras

FIGURA 1. Fases del estudio y variables analizadas en cada etapa

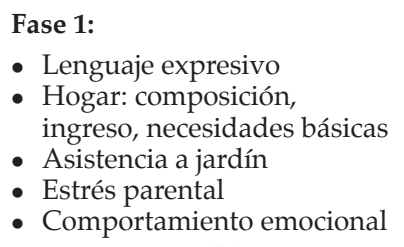
ingreso, necesidades básicas

- Asistencia a jardín

- Estrés parental

- Comportamiento emocional

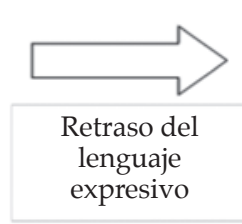

Fase 2:

- Lenguaje receptivo

- Habilidades cognitivas no verbales

- Pesquisa de TEA

- Patología de oído medio

- Audición

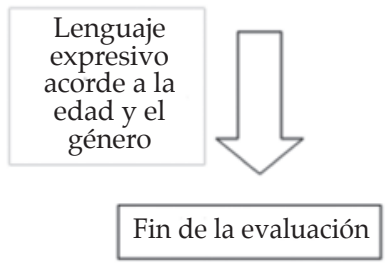

TEA: trastornos del espectro autista. 
fueron adaptadas culturalmente al español local durante la fase piloto, con la autorización de los autores originales del instrumento, respetando el sentido de la palabra original. Por ejemplo, se utilizó fósforos en lugar de cerillos o arveja en lugar de chícharo. Este mismo procedimiento se ha utilizado previamente en versiones en inglés. ${ }^{7,12} \mathrm{El}$ investigador presente actuó como facilitador, según se describía en el manual de uso del instrumento. Se utilizaron las normas de referencia para cada sexo y edad, que establecían la presencia del retraso del lenguaje con puntajes por debajo del percentil 10. Si bien el S-CDI SF-II también incluía la evaluación de la capacidad de combinar palabras, no se utilizó por la falta de disponibilidad de los valores de referencia al momento de iniciar el estudio.

Las variables independientes registradas dentro de la fase 1 mediante cuestionario fueron el sexo, la cantidad de convivientes adultos y de hermanos menores de 18 años, el nivel de educación formal materno (según si completó el nivel secundario o no), el nivel socioeconómico, que medía el ingreso del hogar (indigente, pobre o no pobre, según datos oficiales), ${ }^{13} \mathrm{y}$ las necesidades básicas insatisfechas (NBI) (hogares con hacinamiento, vivienda inadecuada, sin inodoro, niños en edad escolar no escolarizados o de 4 o más integrantes por miembro ocupado con un jefe del hogar con menos de 3 años de primaria), ${ }^{14}$ la asistencia al jardín (en meses) y el antecedente de retraso del lenguaje en los familiares de primer grado. También se indagó sobre el nivel de estrés relacionado con la función parental, mediante la versión corta en español del Parenting Stress Index $(P S I)$, un instrumento validado en España ${ }^{15,16} \mathrm{y}$ utilizado previamente en Argentina ${ }^{17} \mathrm{y}$ en otros trabajos sobre el retraso del lenguaje. ${ }^{5,18}$ Según el puntaje total, se categorizó el resultado como normal o estrés clínicamente significativo. Para indagar sobre problemas del comportamiento y emocionales, se utilizó la versión en español del Childhood Behavior Checklist (CBCL) para niños de entre 1,5 y 5 años, ${ }^{19}$ validada en Argentina ${ }^{20}$ y también empleada en trabajos sobre el retraso del lenguaje. ${ }^{21}$ Según el puntaje total, se categorizó el resultado como normal o problemático (dentro del rango clínico). Cada participante fue evaluado por un único investigador integrante del grupo de 4 pediatras previamente capacitados en la administración de los instrumentos citados y en la recolección de datos.

Todos los participantes que presentaron retraso del lenguaje en la fase 1 fueron posteriormente evaluados en la fase 2. El objetivo de esta segunda fase fue identificar el compromiso del vocabulario receptivo y la posibilidad de causas subyacentes del retraso. El vocabulario receptivo fue estudiado mediante el Inventario de Desarrollo de Habilidades Comunicativas MacArthur-Bates. Palabras y Gestos, versión breve (adaptación argentina) (Spanish-language MacArthur-Bates Communicative Development Inventories short form I, S-CDI SF-I). ${ }^{11}$ En este instrumento autoadministrado, uno de los padres identificó, en una lista de 104 palabras, aquellas que el niño comprendía, y se tomó como lenguaje típico si superaba el percentilo 50 para 18 meses (edad máxima de normas del instrumento). Para esta versión, también con autorización de los autores originales, se adaptaron culturalmente durante la fase piloto 11 palabras a sus equivalentes en español local. Otra versión corta al español se validó en Chile y presentó propiedades psicométricas adecuadas. ${ }^{22}$

Las habilidades cognitivas no verbales se investigaron mediante la versión en español del Cognitive Adaptive Test/Clinical Linguistic and Auditory Milestone Scale (CAT/CLAMS), ${ }^{23}$ recomendada por el Ministerio de Salud de la Nación ${ }^{24}$ y utilizada localmente..$^{25}$ Los resultados podían ser "normal", "sospechoso" o "en rango patológico" (lo cual sugería retraso global del desarrollo). La posibilidad de retraso del lenguaje secundario a TEA fue explorada a través de la versión en español del Modified Checklist for Autism in Toddlers-Revised with Follow up $(M-C H A T-R / F),{ }^{26}$ validada en Argentina. ${ }^{27}$ Los 3 instrumentos de fase 2 fueron administrados por el investigador principal del estudio.

Para el examen de oídos, los participantes fueron estudiados por un especialista en Otorrinolaringología en el Hospital General de Agudos "Parmenio Piñero" mediante microotoscopía binocular, instrumento de alta sensibilidad en la detección de patologías otológicas. ${ }^{28} \mathrm{El}$ examen de audición fue llevado a cabo mediante audiometría tonal por juego (Audiómetro Kamplex® AD 229) en el Hospital de Rehabilitación "Manuel Rocca". En caso de dificultades con la colaboración del participante, se realizó una audiometría en campo libre o por refuerzo visual. No se utilizó como punto de corte el umbral de hipoacusia mínima, sino que se adoptaron los criterios utilizados en la validación de la Prueba Nacional de Pesquisa, ${ }^{29}$ que consideró patológico al participante que presentara como mínimo hipoacusia leve (pérdida 
de 15 a $30 \mathrm{~dB}$ ) de un oído y moderada (pérdida de 30 a $60 \mathrm{~dB}$ ) del otro.

\section{Consideraciones éticas}

Este estudio fue aprobado por el Comité de Ética en Investigación del Hospital General de Agudos "Parmenio Piñero". La madre o el padre de cada participante brindó el consentimiento informado. Se garantizó la confidencialidad de los datos.

\section{Análisis estadístico}

Considerando una prevalencia de retraso del lenguaje de $10-20 \%$ según estudios previos, ${ }^{6,30}$ para un $\alpha=0,1$, se calculó un tamaño muestral de 138 participantes.

Se realizó el análisis univariado, en el que se describieron proporciones con intervalos de confianza (IC) para variables categóricas y medianas con rango intercuartílico para variables numéricas con distribución no normal. Para el análisis bivariado, se emplearon la prueba de chi cuadrado o la prueba de Fisher para la comparación de proporciones (según correspondiera) y la prueba de Mann-Whitney para la comparación entre grupos de variables numéricas de distribución no normal.

Los datos fueron procesados con el programa estadístico Stata 11.2 (Statacorp LP ${ }^{\circledR} 2009$, TX, USA).

\section{RESULTADOS}

Se incluyeron 138 participantes de 994 candidatos. La principal razón para no participar fue la imposibilidad de contactar al candidato (dato telefónico ausente o incorrecto). En los candidatos contactados, los dos principales criterios de exclusión fueron el hogar bilingüe y el nacimiento pretérmino, y solo 2 candidatos rechazaron la invitación. Ningún participante fue excluido por hipoacusia ni problemas del desarrollo previamente diagnosticados. Las respuestas fueron suministradas por la madre en 136 casos $(98,5 \%)$.

Las características de los participantes se describen en la Tabla 1. Más de la mitad fueron varones, y un tercio $(n=46 ; 33 \%)$ de los participantes no tenía hermanos convivientes. Según el ingreso del hogar, 30 (22\%) vivían en hogares indigentes; 93 (67\%), en hogares pobres, y $15(11 \%)$, en hogares no pobres. En cuanto a la asistencia al jardín, dos tercios de los participantes $(n=91 ; 66 \%)$ nunca concurrieron, mientras que aquellos que sí lo hicieron presentaron una mediana de asistencia de 4 meses (rango intercuartílico: 3-10).

Se identificó el retraso del desarrollo del lenguaje en 16 de los 138 participantes (11,6\%; IC 95\%: 6,2$17 \%$ ). La prevalencia de retraso del lenguaje fue 5 veces mayor si existía antecedente familiar de retraso y 4 veces mayor en caso de comportamiento problemático según el CBCL. También se observaron diferencias estadísticamente significativas respecto de los participantes con estrés parental, si bien la magnitud de asociación fue menor (razón de prevalencia-RP-2,6; IC 95\%: 1-6,5). En la fase 2, el compromiso receptivo se observó en 13 de los 16 casos. 4 participantes presentaron habilidades cognitivas no verbales sospechosas de patología y 1 en rango patológico. A su vez, 4 participantes presentaron sospecha de TEA. El examen del oído medio se realizó en 9 participantes, de los cuales 4 fueron normales, 4 presentaron otitis media con derrame bilateral y 1 , retracción timpánica unilateral. Finalmente, se realizó una audiometría en los 9 participantes y fue normal en todos los casos.

TABla 1. Resultados de la evaluación inicial (fase 1, según la presencia de retraso del desarrollo del lenguaje. n=138)

\begin{tabular}{|c|c|c|c|c|c|}
\hline & $\begin{array}{c}\text { Total } \\
(\mathrm{n}=138)\end{array}$ & $\begin{array}{l}\text { Lenguaje típico } \\
\qquad(n=122)\end{array}$ & $\begin{array}{l}\text { Retraso del lenguaje } \\
\qquad(\mathrm{n}=16)\end{array}$ & $\begin{array}{c}\text { RP } \\
(\text { IC 95\%) }\end{array}$ & $p$ \\
\hline \multicolumn{6}{|l|}{$\bar{N}(\%)$ o mediana (rango intercuartílico) } \\
\hline Sexo masculino & $76(55)$ & $67(55)$ & $9(56)$ & & 0,9 \\
\hline Madre c/ secundaria completa & $64(46)$ & $56(46)$ & $8(50)$ & & 0,8 \\
\hline Convivientes adultos & $2(2-3)$ & $2(2-3)$ & $2(2-3,5)$ & & 0,6 \\
\hline Convivientes hermanos & $1(1-2)$ & $1(0-2)$ & $1(0-1,5)$ & & 0,8 \\
\hline Pobre o indigente (según el ingreso) & $123(89)$ & $110(90)$ & $13(81)$ & & 0,3 \\
\hline Necesidades básicas insatisfechas (NBI) & $72(52)$ & $66(54)$ & $6(38)$ & & 0,2 \\
\hline Asistencia al jardín (meses) & $0(0-3)$ & $0(0-3)$ & $0(0-1)$ & & 0,7 \\
\hline Antecedente familiar de retraso del lenguaje & $40(29)$ & $30(25)$ & $10(63)$ & $4,1(1,6-10,5)$ & 0,002 \\
\hline Estrés parental & $26(19)$ & $20(16)$ & $6(38)$ & $2,6(1-6,5)$ & 0,04 \\
\hline Comportamiento problemático (en rango clínico) & $6(4)$ & $3(2)$ & $3(19)$ & $5,1(2-13,1)$ & 0,003 \\
\hline
\end{tabular}

RP: razón de prevalencia; IC: intervalo de confianza. 


\section{DISCUSIÓN}

En el presente estudio, se identificó el retraso del desarrollo del lenguaje en aproximadamente 1 de cada 10 niños, proporción equivalente a la de otros trabajos realizados en poblaciones con mayor representación de niveles socioeconómicos medio y alto. ${ }^{5-8}$ Este problema se presenta cotidianamente en la consulta pediátrica, y se ha destacado la importancia de su identificación y evaluación oportunas. ${ }^{31}$

La asociación encontrada entre el retraso del lenguaje y los problemas de comportamiento se ha observado previamente, al igual que el vínculo con el estrés parental y los antecedentes familiares. ${ }^{5,18,21}$ El nexo entre el nivel socioeconómico y el retraso del desarrollo de lenguaje continúa siendo controvertido, $y$, si bien existen pruebas que sustentan esta relación, ${ }^{32}$ nuestros hallazgos sugieren lo contrario, en concordancia con otras publicaciones. ${ }^{5-8}$ Tampoco se observó una asociación entre el retraso del lenguaje y la asistencia al jardín, al igual que otros autores, ${ }^{6}$ aunque se destaca que, en un estudio reciente, sí se evidenció dicha relación. ${ }^{8}$ Tampoco se pudo demostrar la asociación con la cantidad de convivientes (niños o adultos), en contraposición a la teoría de dilución de recursos familiare ${ }^{33} \mathrm{y}$ a la noción de que un mayor número de hermanos influiría positivamente sobre el vocabulario temprano.

Se encontró un compromiso concurrente del vocabulario receptivo en los participantes con retraso del desarrollo del lenguaje. Llama la atención la alta prevalencia en comparación con los datos anteriores que muestran el compromiso en un tercio total de los participantes. Los distintos enfoques para medir el vocabulario receptivo dificultan las comparaciones en este aspecto. ${ }^{30}$

También se destaca que ninguno de los 9 participantes con retraso del lenguaje presentó una patología auditiva según los criterios utilizados, aun aquellos con otitis media con derrame. Si bien se ha propuesto la evaluación formal de la audición en todos los niños con retraso del desarrollo del lenguaje, ${ }^{10}$ la estrategia óptima de selección de candidatos para la audiometría requiere estudiarse con mayor profundidad, utilizando diseños adecuados a tal fin. Además, a partir de la identificación del retraso del desarrollo del lenguaje, se hallaron casos sospechosos de TEA y de retraso global del desarrollo no detectados previamente, lo que aportó más sustento a la propuesta de pesquisa del lenguaje en la consulta pediátrica de supervisión de salud. Con respecto a esto último, se debe mencionar que, hasta el momento, el grado de evidencia científica sobre los riesgos y los beneficios de pesquisa de retraso del lenguaje en la población general es indeterminado. ${ }^{34}$

Existieron algunas limitaciones en el presente trabajo. Si bien el MacArthur-Bates CDI no fue validado al español en Argentina, otras versiones en español reportaron una validez equivalente a la versión en inglés y otros idiomas. ${ }^{12}$ Aunque los cuestionarios autoadministrados están influenciados por las perspectivas del encuestado, ofrecen la ventaja del conocimiento que este tiene sobre la amplitud del vocabulario del niño en su ámbito natural y por períodos más prolongados, en comparación con el evaluador profesional al que el niño no está familiarizado, en un entorno extraño que puede inhibir sus respuestas. Además, utilizamos el $M-C H A T$ y el CAT/CLAMS, instrumentos que, si bien no ofrecen diagnóstico confirmatorio, poseen sensibilidad y especificidad elevadas en la detección del TEA y del retraso global del desarrollo. ${ }^{23,27} \mathrm{La}$ asociación entre el retraso del lenguaje y las variables estudiadas no pudo ser analizada mediante análisis multivariado debido a limitaciones en el tamaño muestral, por lo que se limitó al análisis bivariado. Finalmente, cerca de la mitad de los participantes con retraso del lenguaje no completaron las evaluaciones de fase 2, lo que evidenció dificultades de accesibilidad y adherencia que también se observaron en otros países. $^{35}$

Entre las principales fortalezas de este trabajo, se resaltan la base poblacional en el primer nivel de atención, el abordaje multidimensional según el modelo ecológico con instrumentos equivalentes a los utilizados en la bibliografía internacional y, en su mayoría, validados localmente, como también la caracterización de los niños y niñas con retraso del lenguaje respecto del compromiso receptivo y posibles causas subyacentes.

La posibilidad de extrapolar los resultados a otros sectores de la población de la CABA puede ser considerada, en particular, a aquellos de estratos socioeconómicos bajos que utilizan los CeSAC para la atención de su salud. Si bien no se indagó sobre el origen étnico de los participantes, el papel de esta característica (considerada en forma aislada del nivel socioeconómico) como determinante del desarrollo del lenguaje es, hasta la actualidad, incierto. ${ }^{8}$ 


\section{CONCLUSIONES}

El retraso del desarrollo del lenguaje representa un problema prevalente en nuestra población y se asoció principalmente con problemas de comportamiento y antecedentes familiares de retraso del lenguaje.

\section{Agradecimientos \\ Donna Jackson-Maldonado por su generosidad y predisposición.}

\section{REFERENCIAS}

1. Feldman HM, Messick C. Language and speech disorders. En: Carey W, Crocker A, Elias E, et al. DevelopmentalBehavioral pediatrics. $4^{\text {th }}$ ed. Philadelphia:Saunders-Elsevier; 2009.Págs.717-29.

2. Bornstein $\mathrm{MH}$, Haynes MO, Painter KM. Sources of child vocabulary competence: a multivariate model. J Child Lang 1998;25(2):367-93.

3. Hawa VV, Spanoudis G. Toddlers with delayed expressive language: An overview of the characteristics, risk factors and language outcomes. Res Dev Disabil 2014;35(2):400-7.

4. Thal D. Late-talking toddlers: Are they at risk? San Diego, CA: San Diego State University; 2000.

5. Horwitz SM, Irwin JR, Briggs-Gowan MJ, et al. Language delay in a community cohort of young children. J Am Acad Child Adolesc Psychiatry 2003;42(8):932-40.

6. Zubrick SR, Taylor CL, Rice ML, et al. Late language emergence at 24 months: an epidemiological study of prevalence, predictors and covariates. J Speech Lang Hear Res 2007;50(6):1562-92.

7. Reilly S, Bavin EL, Bretherton L, et al. The Early Language in Victoria Study (ELVS): A prospective, longitudinal study of communication skills and expressive vocabulary development at 8, 12 and 24 months. Int J Speech Lang Pathol 2009;11(5):344-57.

8. Collisson BA, Graham SA, Preston JL, et al. Risk and protective factors for late talking: An epidemiologic investigation. J Pediatr 2016;172:168-74.

9. Rescorla L. Late talkers: Do good predictors of outcome exist? Dev Disabil Res Rev 2011;17(2):141-50.

10. Buschmann A, Jooss B, Rupp A, et al. Children with developmental language delay at 24 months of age: results of a diagnostic work-up. Dev Med Child Neurol 2008;50(3): 223-9.

11. Jackson-Maldonado D, Marchman VA, Fernald LCH. Short-form versions of the Spanish MacArthurBates Communicative Development Inventories. Appl Psycholinguist 2013;34(4):837-68.

12. Dale PS, Penfold M. Adaptations of the MacArthur $\square$ Bates CDI Into Non $\square$ U.S. English Languages. 2011. [Acceso: 8 de marzo de 2017]. Disponible en: http: / / mb-cdi.stanford. edu/documents / AdaptationsSurvey2011.pdf.

13. Dirección General de Estadística y Censos, Ministerio de Hacienda, Gobierno de la Ciudad de Buenos Aires. Canastas de consumo de la Ciudad de Buenos Aires. Metodología y cálculos iniciales. 2009. [Acceso: 8 de marzo de 2017]. Disponible en: https: / / www.estadisticaciudad. gob.ar/eyc/wp-content/ uploads / 2015/04/canastas_ consumo_2010_enero.pdf.

14. Equipo de trabajo de la Encuesta de Hogares y Empleo. Dirección Provincial de Estadística de la provincia de Buenos Aires. Métodos de Medición de la Pobreza. Conceptos y aplicaciones en América Latina. Entrelíneas de la Política Económica 2010;26(4):31-41. [Acceso: 8 de marzo de 2017]. Disponible en:http:/ / sedici.unlp.edu.ar/ bitstream/handle/10915/15399/Documento_completo. pdf? sequence $=1$.

15. Abidin RR. Parenting Stress Index (PSI) manual. $3^{\text {rd }}$ ed. Charlottesville, VA: Pediatric Psychology Press; 1995.

16. Díaz-Herrero A, López Pina JA, Pérez-López J, etal. Validity of the Parenting Stress Index-short form in a sample of
Spanish fathers. Span J Psychol 2011;14(2):990-7.

17. Basa JA. Estrés parental con hijos autistas. Un estudio comparativo. [Tesis]. Paraná: Universidad Católica Argentina; 2010. [Acceso: 8 de marzo de 2017]. Disponible en:http:/ / bibliotecadigital.uca.edu.ar/repositorio/tesis / estres-parental-con-hijos-autistas.pdf.

18. Irwin JR, Carter AS, Briggs-Gowan MJ. The social-emotional development of "late-talking" toddlers. J Am Acad Child Adolesc Psychiatry 2002;41(11):1324-32.

19. Achenbach TM, Rescorla LS. Manual for the ASEBA Preschool Forms \& Profiles. Burlington, VT: University of Vermont, Research Center for Children, Youth \& Families; 2000.

20. VázquezN,Samaniego VC. ElChild BehaviorChecklist para niños en edad preescolar (CBCL 11/2-5): su estandarización en población urbana de argentina. Trabajo presentado en el VI Congreso Internacional de Investigación y Práctica Profesional en Psicología, XXI Jornadas de Investigación Décimo Encuentro de Investigación en Psicología del MERCOSUR; 27 a 29 de noviembre de 2014. Buenos Aires, Universidad de Buenos Aires; 2014.

21. Carson DK, Klee T, Perry CK, et al. Comparisons of children with delayed and normal language at 24 months of age on measures of behavioral difficulties, social and cognitive development. Infant Ment Health J 1998;19(1):59-75.

22. Farkas Ch. Inventario del desarrollo de habilidades comunicativas MacArthur-Bates (CDI): propuesta de una versión abreviada. Univ Psychol 2011;10(1):245-62.

23. Accardo PJ, Capute AJ. The Capute scales: Cognitive Adaptive Test/Clinical Linguistic \& Auditory Milestone Scale(CAT / CLAMS). Baltimore: Brookes Publishing;2005.

24. Couceiro C, Escandar A, Novali L, et al. Evaluación del neurodesarrollo. En: Unidad Coordinadora Ejecutora de Programas Materno Infantiles y Nutricionales, Ministerio de Salud de la Nación. Guía de Seguimiento del Recién Nacido de Riesgo. 2001: 66-92. [Acceso: 10 de diciembre de 2014]. Disponible en: http:/ / docshare01.docshare.tips / files / 18704/187040359.pdf.

25. Lejarraga $\mathrm{H}$, Berardi $\mathrm{C}$, Ortale $\mathrm{S}$, et al. Crecimiento, desarrollo, integración social y prácticas de crianza en niños que viven con sus madres en prisión. Arch Argent Pediatr 2011;109(6):485-91.

26. Robins D, Fein D, Barton M. Lista de Chequeo Modificada para Deambuladores, Revisada con Seguimiento. 2009. [Acceso: 8 de marzo de 2017]. Disponible en: http:// mchatscreen.com/ wp-content / uploads / 2015/05/MCHAT-R_F_Spanish_Argentina_Uruguay.pdf.

27. Manzone LA. Adaptación y validación del Modified Checklist for Autism in Toddlers para población urbana argentina. Psicodebate 2013;13:79-105.

28. Rogers DJ, Boseley ME, Adams MT, et al. Prospective comparison of handheld pneumatic otoscopy, binocular microscopy, and tympanometry in identifying middle ear effusions in children. Int J Pediatr Otorhinolaryngol 2010;74(10):1140-3.

29. Pascucci MC, Lejarraga H, Kelmansky D, etal. Validación de la prueba nacional de pesquisa de trastornos de desarrollo psicomotor en niños menores de 6 años. Arch Argent Pediatr 2002;100(5):374-84.

30. Desmarais C, Sylvestre A, Meyer F, et al. Systematic review of the literature on characteristics of late-talking toddlers. Int J Lang Commun Disord 2008;43(4):361-89.

31. Camarasa A. Trastornos de la comunicación y el lenguaje: detección temprana. En Sociedad Argentina de Pediatría. PRONAP; 3, 2010.Págs.58-80.

32. Hoff E. The specificity of environmental influence: Socioeconomic status affects early vocabulary development via maternal speech. Child Dev 2003;74(5):1368-78.

33. Downey DB. Number of siblings and intellectual development. Am Psychol 2001;56(6-7):497-504.

34. Siu AL. Screening for speech and language delay and disordersin children aged 5 years or younger: USPreventive Services Task Force recommendation statement. Pediatrics 2015;136(2):e474-81.

35. Brown CM, Beck AF, Steuerwald W, et al. Narrowing care gaps for early language delay: A quality improvement study. Clin Pediatr (Phila) 2016;55(2):137-44. 